\title{
Clinical effect and safety of pulsed radiofrequency treatment for pudendal neuralgia: a prospective, randomized controlled clinical trial
}

This article was published in the following Dove Press journal: Journal of Pain Research

\author{
Hongwei Fang ${ }^{1,2, *}$ \\ Jinyuan Zhang ${ }^{2, *}$ \\ Yu Yang ${ }^{1, *}$ \\ Le $\mathrm{Ye}^{2}$ \\ Xiangrui Wang'
}

'Department of Anesthesiology and Intensive Care Unit, Dongfang Hospital, Tongji University, Shanghai, China; ${ }^{2}$ Department of Pain Management, South Campus, Renji Hospital, School of Medicine, Shanghai Jiao Tong University, Shanghai, China

*These authors contributed equally to this work

Correspondence: Xiangrui Wang Department of Anesthesiology and Intensive Care Unit, Dongfang Hospital, Tongji University, No. 150 Jimo Road, Shanghai 200120, China Email xiangrui68@I63.com
Background: Pudendal neuralgia is an intractable pain related to the pudendal nerve. The clinical effect and safety evaluation of pudendal neuralgia were investigated by pulse radiofrequency (PRF) treatment of pudendal nerve.

Patients and methods: Eighty patients who were diagnosed with pudendal neuralgia were randomly divided into PRF group (PRF and pudendal nerve block [NB]) and NB group. After surgery, the patients were followed up to evaluate the visual analog scale (VAS) score and the Patient Health Questionnaire score on the postoperative day and at 2 weeks, 1 and 3 months. Meanwhile, the patients' efficacy assessment and the usage of pain medication were also recorded for 3 months during follow-up. All the surgical complications were recorded.

Results: A total of 77 patients were followed up, 38 in the PRF group and 39 in the NB group. On the postoperative day, the VAS scores was significantly decreased in both groups than before $(P<0.01)$, whereas there was no statistical difference within the two groups $(P>0.05)$. However, the VAS score of PRF group was significantly lower than that of NB group in 2 weeks, 1 and 3 months after surgery, respectively $(P<0.01)$. In the meanwhile, the Patient Health Questionnaire score of PRF group was also significantly lower than that of NB group $(P<0.01)$ in 3 months after the operation. The clinical effective rate of PRF group was $92.1 \%$ in 3 months after surgery, while this rate was only $35.9 \%$ in the NB group. The postoperative analgesic usage of PRF group was superior to that of NB group $(P<0.01)$. No severe adverse events were observed in either group.

Conclusion: Compared with the single NB treatment, pudendal nerve PRF combined with NB therapy could provide more long-lasting relief from pain symptoms of pudendal neuralgia and improve the depression symptoms in patients.

Keywords: pudendal neuralgia, pulse radiofrequency treatment, pudendal nerve block, ultrasound guidance, visual analog scale, PHQ-9

\section{Introduction}

Pudendal neuralgia is an illness which involves neuropathy of the pudendal nerve. According to the International Association of Pudendal Neuropathy, the incidence of pudendal neuralgia in the general population is about $1 / 100,000 .{ }^{1}$ Pudendal neuralgia is caused by inflammation, compression and traction of the pudendal nerve. It may be associated with childbirth, pelvic surgery, strenuous exercise, abnormalities of the sacroiliac joint, and also, it is connected to age-related changes. ${ }^{2}$ The clinical features of this disease include hyperalgesia in the perineal region during sitting, which becomes worse during the day and could be relieved by standing or lying position, and the 
disease is associated with sexual dysfunction and difficulties in urination and/or defecation. The feeling of hyperalgesia can be felt in the vagina or rectum with elevated fever, swelling and/or foreign body sensation. Pudendal neuralgia is an intractable pain related to the pudendal nerve, which severely affects the quality of life and is hard to treat. ${ }^{3,4}$

Currently, the clinical treatments of pudendal neuralgia include drug therapy, pudendal nerve block (NB), pudendal nerve decompression, nervous regulation by the implanted pulse generator, radiofrequency ablation, spinal cord electrical stimulation and so on. Continuous radiofrequency ablation (CRF) has been used in clinical practice for $>25$ years as a pain treatment by using the minimally invasive percutaneous techniques. This method has a large number of advantages such as fewer complications, more accurate positioning, less adverse effects and lower recurrence rate. Indeed, CRF is now one of the most important methods for pain relief and it is widely used in clinical practice. ${ }^{5}$ Pulse radiofrequency (PRF) is a new type of neuromodulation technique which is safer than conventional CRF. ${ }^{6}$ Although the specific mechanism of PRF is unclear, the current literature supports that the intervention of electromagnetic fields achieves neuromodulation.

The results of preclinical treatment in our research group and recent literature suggest that PRF may be effective for treatment of refractory neuropathic pain. In this study, we researched the clinical efficacy and safety of two therapeutic regimens for the treatment of patients with pudendal neuralgia.

\section{Patients and methods General information}

The study was approved by the ethical review committee of Renji Hospital. This study is a prospective, randomized controlled clinical trial. The subjects were recruited from patients who received treatment in the pain department of Renji Hospital from January 2015 to September 2017. At recruitment, written informed consent was obtained from each subject.

\section{Inclusion criteria}

According to the Nantes criteria, ${ }^{7}$ the essential diagnostic criteria of pudendal neuralgia include the following: 1) pain located in the pudendal nerve distribution area; 2) pain intensity increases in the sitting position; 3 ) no nocturnal pain symptoms; 4) pain with no objective sensory impairment and 5) pain is relieved by diagnostic pudendal NB. Another clinical standard provides extra support to the diagnosis of pudendal neuralgia: 6) the pain symptom is associated with sexual dysfunction, with the elimination of obstetrical and gynecologic, urological and anorectal diseases in the related departments and absence of any physical and mental disorders; 7) treatment by other departments is invalid; ${ }^{8}$ patients should be older than 18 years and 9) patients should be able to sign an informed consent form.

\section{Exclusion criteria}

The exclusion criteria for pudendal neuralgia include the following: 1) patients have simple tailbone, gluteus muscle or lower abdominal pain, or only paroxysmal pain, or only pruritic symptoms, and/or have imaging anomalies that may explain the symptoms; 2) female patients who are pregnant; 3 ) patients who take anticoagulant drugs or have any coagulation disorder; 4) patients who are unable to complete the questionnaire; 5) patients with pain, which is caused by malignant or autoimmune diseases and 6) patients who are hypersensitive to metals.

\section{Test group}

A random assignment sequence was generated by the computer, and 80 patients with pudendal neuralgia were randomly assigned and divided into two groups, that is, the PRF+NB group and the NB group. Each group contained 40 patients at the beginning of the clinical trial.

\section{Therapeutic schedule}

The patients were treated as follows. The patient was made to lie in a prone position and a thin pillow was placed under the patient's anterior lower abdomen and the projection area of the pudendal nerve on the body surface was labeled. Thereafter, the skin was sterilized by povidone iodine and a sterile surgical towel was placed on the patient (Figure 1A). The areas were scanned and marked by low-resolution (12-16 $\mathrm{mHz}$ ) linear array transducer probe (S-Nerve; Sonosite, Bothell, WA, USA). First, in order to identify the bone sign of the ischial spine, an ultrasound transducer was placed at the level of the sacrospinous ligament and the internal iliac artery and the pudendal nerve position were identified (Figure 1B). Then, local anesthesia was administered by skin infiltration of $1 \%$ lidocaine. Second, under the guidance of ultrasound (S-Nerve, Sonosite), the $20 \mathrm{G}, 10 \mathrm{~cm}$ puncture needle with $5 \mathrm{~mm}$ tip (RF Simject Cannula; NeuroTherm, Wilmington, MA, USA) was inserted near the pudendal nerve (Figure 1C). On entering into the visual operating area, the surgeon should pay attention to the patient's response (the patient's painful response appears in the same position, but is more severe 


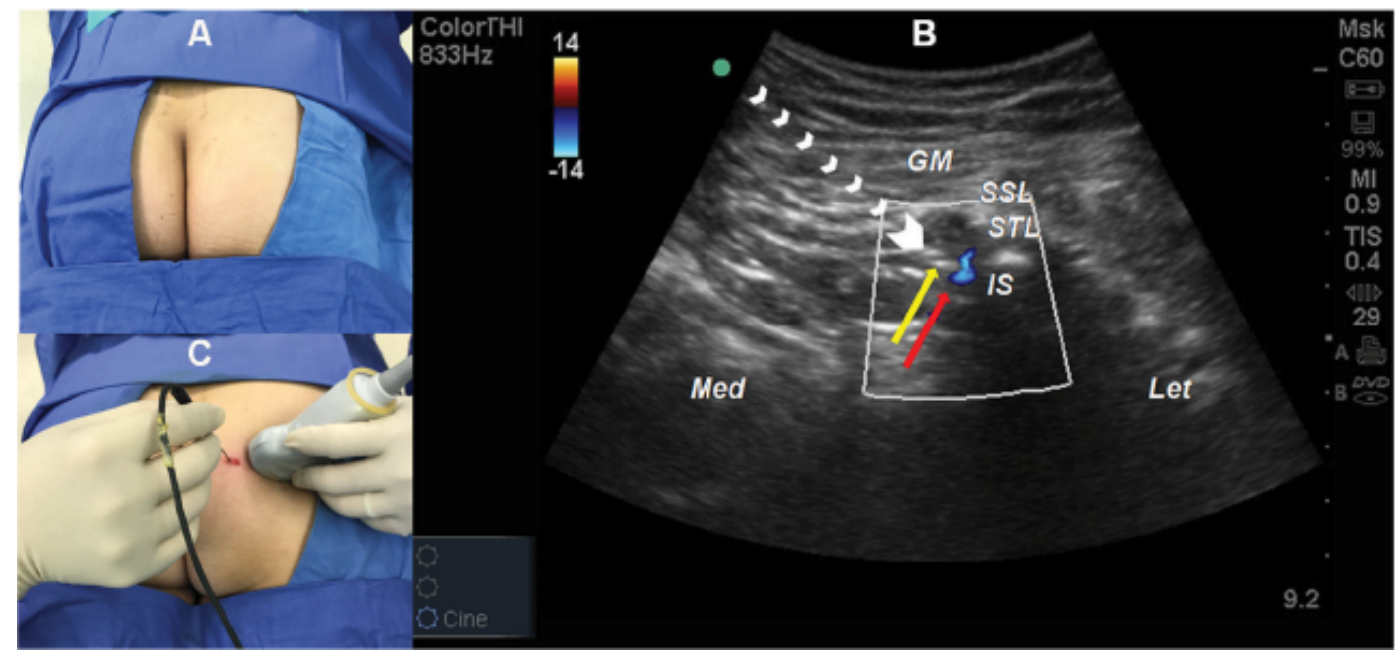

Figure I Pudendal nerve NB or PRF treatment diagram.

Notes: (A) Patient is in prone position and a thin pillow is placed under the patient's anterior lower abdomen. Then the skin is sterilized by povidone iodine and a sterile surgical towel is placed on the patient. (B) Ultrasonic positioning on the right side of pudendal nerve (yellow arrow), pudendal artery (red arrow pointing to the blue area), dotted line shows the route of the puncture needle. (C) Puncture needle is located around the pudendal nerve; then, the doctor proceeds with the NB or PRF treatment. Abbreviations: GM, glutes; IL, ischial spine; NB, nerve block; PRF, pulse radiofrequency; SSL, the nodule ligaments, STL, sacral spine ligaments.

than usual). Later, the surgeon proceeded with the treatment according to the protocol of the two groups.

\section{NB group}

After pulling out the needle core, the surgeon injected 5 $\mathrm{mL}$ of the nutrient nerve compound (compound liquid 20 $\mathrm{mL}=$ Neurotropin ${ }^{\circledR} 6 \mathrm{~mL}+2 \%$ lidocaine $5 \mathrm{~mL}+0.75 \%$ ropivacaine $2 \mathrm{~mL}+0.9 \% \mathrm{NaCl} 7 \mathrm{~mL}$ ). Then, the surgeon removed the puncture needle and compressed this area for 3 minutes for hemostasis. The puncture point should be covered with sterile material for 3 days to prevent any infection.

\section{PRF combination with NB group}

After connecting the electrode and the PRF needle, the surgeon determined whether the pudendal nerve innervation produced paresthesia in the sensory stimulation test with a frequency of $50 \mathrm{~Hz}$, pulse width of $1 \mathrm{~ms}$ and a voltage of $0.3-0.5 \mathrm{~V}$. Thereafter, the radiofrequency ablation therapy was performed in $42^{\circ} \mathrm{C}, 120$ seconds pulse mode (PMG230 Baylis Medical Company Inc., Montreal, QC, Canada), which consisted of two cycles. Following that, the surgeon injected 5 $\mathrm{mL}$ of the nerve nutrition compound liquid through radiofrequency trocar after pulling out the radiofrequency electrode. Finally, the surgeon removed the puncture needle. The other care treatment was the same as in the former group.

We defined 24-48 hours after surgery as the first postoperative day (POD). All patients returned to the ward after the operation and received the minimum oral dose of pregabalin (one tablet twice daily after meals) as a routine pain treatment from POD, in order to obtain relief from pain and to control the visual analog scale (VAS) score below four points. When patients had a breakthrough pain, they were recommended to relieve the pain by taking one oxycodone-acetaminophen tablet. All treatments were performed by the same researcher, while the follow-up visit and evaluation were done by other researchers.

\section{Assessment criteria}

1. Pain assessment: The VAS score was used that has $0-10$ points. The 0 point represents the painless state and a score from 1 to 3 points represents mild pain, from 4 to 6 points represents moderate pain and from 7 to 10 points represents severe pain. The VAS evaluation was performed before the operation and on the POD and at 2 weeks, 1 and 3 months after the operation.

2. Evaluation of treatment effects: The treatment effects were evaluated by the VAS score, pain symptoms and local physical signs. The treatment effect was divided into four grades - "completely cured", "significant positive effect", "effective" and "invalid". The "completely cured" grade indicates that the symptoms and physical signs of the disease disappear, restoring a good quality of life. The "significant positive effect" grade is given when the symptoms are significantly alleviated and the quality of life is improved, but there is still intermittent tolerable pain. The "effective" grade is given when the symptoms are alleviated, but the effect is not permanent and does 
not last for a long time. The "invalid" grade is given when the symptoms are not improved and the quality of life is the same as before. The "effective rate" was estimated by the number of [(cured + significant effect + effective $) /$ total number] $\times 100 \%$.

3. Depression assessment: The Patient Health Questionnaire (PHQ-9; depression table) was used to measure the anxiety-depression degree in patients. PHQ-9 is a reliable and valid measure of depression severity and is scored as follows: ${ }^{8}$ from 5 to 10 points was considered to be mild depression, from 10 to 15 points as moderate depression, from 15 to 20 points as moderately severe depression and $>20$ points as severe depression. Depression assessment was performed only at the final follow-up visit.

4. Postoperative analgesic usage: The time that the pain lasted and the number of people who used oxycodoneacetaminophen tablet to be relieved from acute pain were recorded during the follow-up visit.

5. Adverse events: Participants were enquired at each follow-up visit if they had any adverse reactions and accident medical events during the period from the last visit, including hypertension or hypotension, dizziness, nausea, vomiting, lower limb numbness, urinary retention, puncture point of hematoma and so on. The doctors recorded every adverse event and reported them on time.

\section{Statistical analysis}

Data on continuous variables conforming to normal distribution were presented as mean $\pm \mathrm{SD}$; otherwise, data were presented as median (range). All data including the VAS and PHQ-9 scores after the treatment were compared with the baseline by paired $t$-test. Intergroup comparisons were performed by repeated measure analysis of variance for normally distributed data. Chi-squared test was used for comparing the categorical variables between the two groups. Non-normal distribution of measurement data and ranked data were compared by the Mann-Whitney nonparametric tests. Statistical software SPSS 22.0 was used for data statistical analysis. $P<0.05$ was considered to be statistically significant.

\section{Results}

A total of 77 patients completed the entire 3 months followup. The NB group contained 39 patients (1 person was lost during the follow-up period) with an average age of $45 \pm 15.1$ years, an average body mass index of $25.4 \pm 3.9$ and an average treatment duration of $7.4 \pm 3.9$ months. The PRF group contained 38 patients ( 1 person was lost during the follow-up period and 1 person refused to participate in the next visits) with an average age of $45.1 \pm 12.6$ years, an average body mass index of $23.3 \pm 3$ and an average duration of $6.4 \pm 3.8$ months (Figure 2; Table 1).

The effect of treatment was evaluated by comparison of VAS score at each time point between the two groups (Figure 3). There was no difference in the VAS score between the two groups preoperatively. On the POD, the VAS score decreased significantly in both the groups than before the treatment $(P<0.01)$, without any difference between the two groups $(P>0.05)$. After 2 weeks, 1 and 3 months, the VAS score decreased more significantly in the PRF group than in the NB group $(P<0.01$; Table 2$)$.

The PHQ-9 scale was used to measure the anxietydepression degree in the two groups of patients before and after the operation (Table 2). There was no difference between the two groups of patients preoperatively $(P>0.05)$. After 3 months of operation, the PHQ-9 score decreased significantly in the PRF group compared with the NB group $(P<0.01)$.

The comparison of effect between the two groups is shown in Table 2. The "completely cured", "significant effect" and "effective" were marked as "effective". Three months after the procedure, the effective rate was $92.1 \%$ in the PRF group, but only $35.9 \%$ in the NB group. The comparison between groups was performed by the Mann-Whitney nonparametric test. The effect of PRF group was considerably better than of NB group.

The analgesic usage is shown in Table 3. All patients in the NB group took pregabalin for 3 months after surgery except 1 who stopped taking it 2 months postprocedure, while in the PRF group, 5 patients took the drug for 1 month, 5 patients for 2 months and 28 patients for 3 months. The number of patients taking analgesics (oxycodone and acetaminophen tablets) after surgery was recorded and statistically analyzed. Twenty-one patients $(53.8 \%)$ in the NB group took the analgesic for analgesia postoperatively, whereas in the PRF group, only nine patients (23.7\%) took the analgesic. The analgesic effect in the PRF group was significantly better than in the NB group $(P<0.01)$.

During the 3-month follow-up, of the total 77 patients, in the NB group, only 1 patient had hypertension and 2 patients had dizziness. Meanwhile, one patient had hypertension and one patient had dizziness in the PRF group. No severe adverse events had occurred in both groups during the follow-up period.

\section{Discussion}

These experiments compared the therapeutic effects of pudendal NB treatment and PRF of the pudendal nerve with NB treatment in patients with pudendal neuralgia. Patients who completed the follow-up $(\mathrm{N}=77)$ were divided into two 


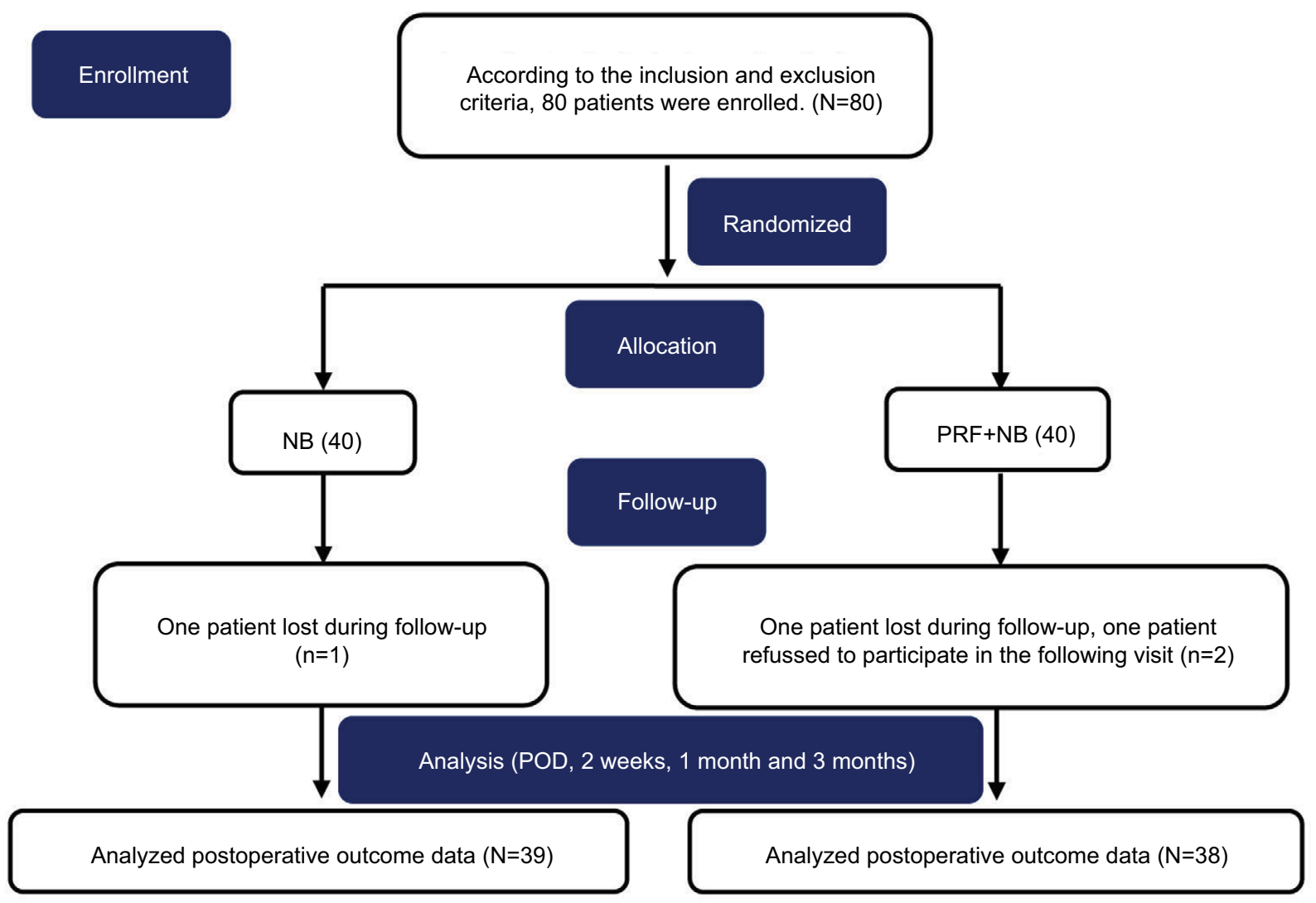

Figure 2 Consort flow diagram.

Abbreviations: NB, nerve block; PRF, pulse radiofrequency; POD, postoperative day.

Table I Baseline characteristics of the patients

\begin{tabular}{llll}
\hline Characteristic & NB $(\mathbf{n = 3 9 )}$ & PRF $(\mathbf{n = 3 8 )}$ & P-value \\
\hline Age (years) & $\mathbf{4 5 \pm I 5 . I}$ & $\mathbf{4 5 . I \pm I 2 . 6}$ & $\mathbf{0 . 9 6 7}$ \\
BMI & $25.4 \pm 3.9$ & $23.3 \pm 3$ & 0.011 \\
Duration of pain (months) & $7.4 \pm 3.9$ & $6.4 \pm 3.8$ & 0.257 \\
Baseline VAS score & $5.5 \pm I .4$ & $5.7 \pm I .3$ & 0.631 \\
Liver and renal function & $0 / 38$ & $0 / 39$ & \\
abnormal & & & \\
Pain character & & & \\
$\quad$ Stabbing pain & 10 & 7 & 0.987 \\
$\quad$ Burning pain & 19 & 20 & \\
$\quad$ Aching pain & 5 & 7 & \\
$\quad$ Throbbing pain & 5 & 4 & \\
\hline
\end{tabular}

Notes: Data for continuous variables are reported as the mean $\pm S D$. Data for categorical variables are reported as \%. Independent Student's t-test was used for normally distributed data and chi-squared test for comparing the categorical variables between the two groups. Bold figures indicate that the age of the two groups is statistically significant based on actual data, but it cannot represent any significance in clinical work and has no impact on our clinical research results.

Abbreviations: BMI, body mass index; NB, nerve block; PRF, pulse radiofrequency; VAS, visual analog scale.

groups based on multiple assessment criteria including postoperative VAS score, preoperative treatment curative effect, depression scale score, postoperative pain, analgesic usage and the incidence of postoperative complications. As shown in Figure 3, the VAS score in both groups was decreased on the first day after the operation compared with the preoperative score. Interestingly, the VAS scores at 2 weeks and 1 and 3 months postoperatively were significantly lower in the PRF group than in the NB group $(P<0.01)$. The clinical effect was significantly better in the PRF group than in the NB group. Similarly, the PRF group had lower PHQ-9 score than the NB group 3 months after the operation $(P<0.01)$. No severe postoperative complications were found in either group.

The relatively complex anatomical structure of the pelvis has been a focus of study in the medical field. The diagnosis of the causes of chronic pelvic pain is usually a complicated process. ${ }^{9}$ Pudendal nerve is a mixed nerve which contains sensory as well as motor functions and consists of S2, S3 and $\mathrm{S} 4$ nerves. These nerves originate from the sacral plexus and dominate in the areas of the anus, urethral sphincter, pelvic floor and perineum. At the same time, they regulate the sensitivity of the genitals. ${ }^{6}$ Pudendal neuralgia is a syndrome that is characterized by pelvic pain, including bowel, bladder regional pain or discomfort, sexual dysfunction, severe burning sensations and needle-like pain, which are aggravated when seated and relieved after standing. 


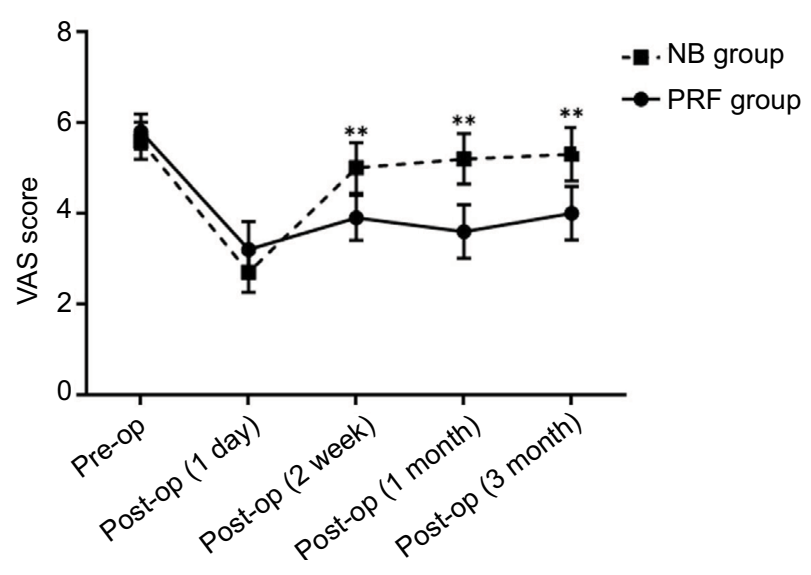

Figure 3 VAS scores of the two groups at each time point.

Note: **VAS scores showed statistically significant difference between the two groups.

Abbreviations: NB, nerve block; PRF, pulse radiofrequency; VAS, visual analog scale.

Table 2 Comparison of primary and secondary outcomes in patients with pudendal neuralgia treated with NB or PRF

\begin{tabular}{|c|c|c|c|}
\hline Outcome & $\begin{array}{l}\text { NB } \\
(n=39)\end{array}$ & $\begin{array}{l}\text { PRF } \\
(n=38)\end{array}$ & P-value \\
\hline \multicolumn{4}{|l|}{ VAS } \\
\hline Preoperation & $5.5 \pm 1.4$ & $5.7 \pm 1.3$ & 0.631 \\
\hline \multicolumn{4}{|l|}{ Postoperation } \\
\hline I Day & $2.6 \pm 1.4$ & $3.1 \pm 2$ & 0.247 \\
\hline 2 Week & $5 \pm 1.9$ & $3.8 \pm 1.8$ & 0.008 \\
\hline I Month & $5.1 \pm 1.8$ & $3.6 \pm 1.9$ & 0.001 \\
\hline 3 Month & $5.2 \pm 1.9$ & $3.9 \pm 2.1$ & 0.004 \\
\hline \multicolumn{4}{|l|}{ PHQ-9 scores } \\
\hline Preoperation & $9.1 \pm 2.1$ & $8.9 \pm 2.1$ & 0.747 \\
\hline Postoperation 3 months & $8.1 \pm 2.4$ & $6.4 \pm 1.8$ & 0.001 \\
\hline \multicolumn{4}{|l|}{$\begin{array}{l}\text { Clinical curative effect } \\
\text { observation }(n)\end{array}$} \\
\hline Heal & I & 3 & $<0.01$ \\
\hline Excellent & 4 & 12 & \\
\hline Effectively & 9 & 20 & \\
\hline Ineffectiveness & 25 & 3 & \\
\hline \multicolumn{4}{|l|}{ Adverse reactions $(n)$} \\
\hline Hypertension & I & I & 0.985 \\
\hline Dizziness & 2 & I & 0.547 \\
\hline
\end{tabular}

Notes: Data for continuous variables are reported as the mean \pm SD if they showed a normal distribution; otherwise, data are reported as median (range). VAS and PHQ9 scores after the treatment were comparable to the baseline using paired $t$-test. Intergroup comparisons were performed by RMANOVA for normally distributed data. Chi-squared test was used for comparison of categorical variables between the two groups. Non-normal distribution of measurement data and ranked data were compared using the Mann-Whitney nonparametric tests. $P<0.05$ was considered to be statistically significant.

Abbreviations: NB, nerve block; PHQ-9, Patient Health Questionnaire; PRF, pulse radiofrequency; RMANOVA, repeated measure analysis of variance; VAS, visual analog scale.

There are many pathologies that can cause pudendal nerve injury lacking sensory or motor symptoms. The oppression of pudendal nerve in the anatomical position is likely to be one of the factors leading to this kind of injury. The majority of
Table 3 Comparison of postoperative analgesic usage in patients with pudendal neuralgia treated with NB or PRF

\begin{tabular}{llll}
\hline Outcome & $\begin{array}{l}\text { NB } \\
(\mathbf{n = 3 9 )}\end{array}$ & $\begin{array}{l}\text { PRF } \\
(\mathbf{n = 3 8 )}\end{array}$ & P-value \\
\hline $\begin{array}{l}\text { Postoperative analgesic usage }(\mathrm{n}) \\
\text { Number of patients taking }\end{array}$ & & & \\
$\begin{array}{l}\text { pregabalin } \\
\text { I Month }\end{array}$ & 0 & 5 & $<0.01$ \\
2 Month & $\mathrm{I}$ & 5 & \\
$\begin{array}{l}\text { Month } \\
\text { Whether oxycodone- }\end{array}$ & 38 & 28 & \\
acetaminophen was taken & & & \\
Yes/no & $2 \mathrm{I} / 18$ & $9 / 29$ & $<0.01$ \\
\hline
\end{tabular}

Notes: We recorded and compared the number of patients who had taken analgesic drugs after surgery in the two groups respectively. Chi-squared test was used for comparing the categorical variables between the two groups. Non-normal distribution of measurement data and ranked data were compared using the MannWhitney nonparametric tests. $P<0.05$ was considered to be statistically significant.

Abbreviations: NB, nerve block; PRF, pulse radiofrequency.

pudendal neuralgias are induced by pudendal nerve damage. ${ }^{10}$ During childbirth, the pudendal nerve may be pulled to the pelvic floor and perineum. Similarly, gynecologic surgery may result in pudendal nerve damage, especially during the treatment of genital prolapse or incontinence via the vagina, pelvic reconstruction, urethra suspension surgery as well as vagina revascularization, among others. ${ }^{11}$

Current treatment of patients with pudendal neuralgia includes drug therapy, NB, pressure release surgery and neuromodulation therapy, and site-specific surgical or transplant approach. ${ }^{12,13}$ Nevertheless, the ideal clinical treatment for pudendal neuralgia has not yet been determined. During clinical treatment, patients should use pudendal NB instead of invalid conservative medical treatment. Filippiadis et al concluded that percutaneous puncture infiltration block of the pudendal nerve is a safe technique for the treatment of pudendal neuralgia. ${ }^{14}$ Additionally, Abdi et al found that under the guidance of $\mathrm{C}$ arm, pudendal NB (3-4 mL local anesthetics) is safe to administer to patients in the prone position and increases patient comfort. ${ }^{15}$ Interestingly, Labat et al demonstrated that use of cortical hormones is not recommended during pudendal NB. ${ }^{7}$ The treatment strategies should include only local infiltration anesthesia, without adding corticosteroids, and confirm the effect of injection in the sacral spine ligament. These authors found $26 \%$ of the patients had a decrease in their pain score $>30 \%$. Currently, pudendal NB is widely used in clinical practice. However, Amarenco et al indicated that after the pudendal NB operation, $57 \%$ of patients had an immediate effect, but during the 12 -month follow-up period, only $15 \%$ of patients had a good long-term effect. ${ }^{16}$ The pudendal NB method could obviously relieve symptoms in a short period of time; 
but because of its short effective period, patients should receive the pudendal NB therapy repeatedly to alleviate the symptoms.

Radiofrequency treatment is gradually being used on pudendal nerve for treatment of chronic pelvic pain in the clinical settings. Currently, two radiofrequency patterns are used in clinical practice - CRF and PRF. In the traditional CRF method, heat is generated by an electrode and focused on the target nerve; it produces a magnetic current and directly causes nerve damage. ${ }^{17}$ In clinical practice, CRF is widely used in the treatment of the following pathological conditions: zygomatic joint osteoarthritis, trigeminal neuralgia and occipital neuralgia. ${ }^{18-21}$ Karaman et al indicated that CRF may alleviate perineal pain, but could lead to bowel, bladder and sexual dysfunction because of the high temperature required..$^{22}$ Therefore, due to its advantage of no irreversible tissue damage by keeping the temperature below $45^{\circ} \mathrm{C}-50^{\circ} \mathrm{C}$, PRF was used as a substitute for traditional CRF. Rhame et al reported that female patients, who suffered from sharp burning pain for 1.5 years, experienced pain relief after PRF treatment $\left(2 \mathrm{~Hz}, 42^{\circ} \mathrm{C}\right.$ and 120 seconds). ${ }^{6}$ Additionally, Ozkan et al discussed the clinical effect of the PRF in three patients with chronic pelvic pain. ${ }^{23}$ Recently, Petrov-Kondratov et al reported on the case of a 51-year-old female with pain in genital area for 5 years, whose pain score decreased $>50 \%$ at 6 weeks postoperatively during the follow-up after PRF $\left(42^{\circ} \mathrm{C}\right.$ and 240 seconds) treatment. ${ }^{24}$

This study compared postoperative VAS score at several time points and found that both PRF and NB could decrease pudendal neuralgia symptoms in patients on POD without any statistical difference between the two groups. However, the VAS score increased gradually after the operation. After 2 weeks and 1 and 3 months postoperatively, the VAS score in the PRF group was significantly lower than in the NB group. Therefore, in a short period of time, both treatments could improve the symptoms of pudendal neuralgia, but the medium and long-term effect in the NB group was worse than in the group receiving PRF with NB. The effective rate in the PRF group was $93 \%$, while it was only $40 \%$ in the NB group at 3 months postoperatively. The usage of analgesic drugs confirmed that there are fewer patients who suffer from breakthrough pain in the PRF group and have better long-term pain control than those in NB group. Finally, 3 months postoperatively, the anxiety-depression degree was significantly lower in the PRF group than in the NB group, confirming that PRF therapy alleviates pudendal nerve pain and improves the patient's quality of life.
The mechanism of PRF has been studied repeatedly. It has been verified that PRF has definite safe treatment range, but during the PRF treatment, the tissue surrounding the electrode may have some degree of change and so it is difficult to assess any actual damage. ${ }^{25}$ Based on electric field intensity, PRF can actually produce tissue changes observable as in vitro changes to ultrastructure in early-phase histologic study. These biological changes were converted to biological effect to alleviate neuropathic pain in animal models.

\section{Conclusion}

The study results suggest that PRF with NB therapy can alleviate pain symptoms of patients with pudendal neuralgia and improve their quality of life by decreasing the depression symptoms for a longer time compared to the effect of only pudendal NB therapy, and that the use of analgesic drugs in the PRF with NB group was lower compared to the use of analgesics in the NB group, without increasing the incidence of postoperative adverse events.

\section{Disclosure}

The authors report no conflicts of interest in this work.

\section{References}

1. Hibner M, Desai N, Robertson LJ, Nour M. Pudendal neuralgia. J Minim Invasive Gynecol. 2010;17(2):148-153.

2. Pérez-López FR, Hita-Contreras F. Management of pudendal neuralgia. Climacteric. 2014;17(6):654-656.

3. Dellon AL, Coady D. Vulvar and pelvic pain terminology review: implications for microsurgeons. Microsurgery. 2015;35(2):85-90.

4. Bornstein J, Goldstein AT, Stockdale CK, et al. Consensus vulvar pain terminology committee of the International Society for the Study of Vulvovaginal Disease (ISSVD), the International Society for the Study of Women's Sexual Health (ISSWSH), and the International Pelvic Pain Society (IPPS). 2015 ISSVD, ISSWSH and IPPS consensus terminology and classification of persistent vulvar pain and vulvodynia. Obstet Gynecol. 2016;127:745-751.

5. Racz GB, Ruiz-Lopez R. Radiofrequency procedures. Pain Pract. 2006;6(1):46-50.

6. Rhame EE, Levey KA, Gharibo CG. Successful treatment of refractory pudendal neuralgia with pulsed radiofrequency. Pain Physician. 2009;12(3):633-638.

7. Labat JJ, Riant T, Robert R, Amarenco G, Lefaucheur JP, Rigaud J. Diagnostic criteria for pudendal neuralgia by pudendal nerve entrapment (Nantes criteria). Neurourol Urodyn. 2008;27(4):306-310.

8. Kroenke K, Spitzer RL, Williams JB. The PHQ-9: validity of a brief depression severity measure. J Gen Intern Med. 2001;16(9):606-613.

9. Benson JT, Griffis K. Pudendal neuralgia, a severe pain syndrome. Am J Obstet Gynecol. 2005;192(5):1663-1668.

10. Báča V, Báčová T, Grill R, et al. Pudendal nerve in pelvic bone fractures. Injury. 2013;44(7):952-956.

11. Pailhé R, Chiron P, Reina N, Cavaignac E, Lafontan V, Laffosse JM. Pudendal nerve neuralgia after hip arthroscopy: retrospective study and literature review. Orthop Traumatol Surg Res. 2013;99(7):785-790.

12. Dellon AL, Coady D, Harris D. Pelvic pain of pudendal nerve origin: surgical outcomes and learning curve lessons. J Reconstr Microsurg. 2015;31(4):283-290. 
13. Wan EL, Goldstein AT, Tolson H, Dellon AL. Injury to perineal branch of pudendal nerve in women: outcome from resection of the perineal branches. J Reconstr Microsurg. 2017;33(6):395-401.

14. Filippiadis DK, Velonakis G, Mazioti A, et al. CT-guided percutaneous infiltration for the treatment of Alcock's neuralgia. Pain Physician. 2011;14(2):211-215.

15. Abdi S, Shenouda P, Patel N, Saini B, Bharat Y, Calvillo O. A novel technique for pudendal nerve block. Pain Physician. 2004;7(3):319-322.

16. Amarenco G, Kerdraon J, Bouju P, et al. Treatments of perineal neuralgia caused by involvement of the pudendal nerve. Rev Neurol. 1997;153(5):331-334.

17. Choi WJ, Hwang SJ, Song JG, et al. Radiofrequency treatment relieves chronic knee osteoarthritis pain: a double-blind randomized controlled trial. Pain. 2011;152(3):481-487.

18. Masala S, Nano G, Mammucari M, Marcia S, Simonetti G. Medial branch neurotomy in low back pain. Neuroradiology. 2012;54(7):737-744.

19. Weyker P, Webb C, Mathew L. Radiofrequency ablation of the supraorbital nerve in the treatment algorithm of hemicrania continua. Pain Physician. 2012;15(5):E719-E724.
20. He YQ, He S, Shen YX, Qian C. A feasibility study on pinpointing the branches of trigeminal nerve in radiofrequency ablation. Br J Neurosurg. 2013;27(2):235-240.

21. Choi HJ, Oh IH, Choi SK, Lim YJ. Clinical outcomes of pulsed radiofrequency neuromodulation for the treatment of occipital neuralgia. J Korean Neurosurg Soc. 2012;51(5):281-285.

22. Karaman H, Tüfek A, Kavak GÖ, et al. Intra-articularly applied pulsed radiofrequency can reduce chronic knee pain in patients with osteoarthritis. J Chin Med Assoc. 2011;74(8):336-340.

23. Ozkan D, Akkaya T, Yildiz S, Comert A. Ultrasound-guided pulsed radiofrequency treatment of the pudendal nerve in chronic pelvic pain. Anaesthesist. 2016;65(2):134-136.

24. Petrov-Kondratov V, Chhabra A, Jones S. Pulsed radiofrequency ablation of pudendal nerve for treatment of a case of refractory pelvic pain. Pain Physician. 2017;20(3):E451-E454.

25. Cahana A. Pulsed radiofrequency: a neurobiologic and clinical reality. Anesthesiology. 2005;103(6):1311; author reply 1313-1314.
Journal of Pain Research

\section{Publish your work in this journal}

The Journal of Pain Research is an international, peer reviewed, open access, online journal that welcomes laboratory and clinical findings in the fields of pain research and the prevention and management of pain. Original research, reviews, symposium reports, hypothesis formation and commentaries are all considered for publication.

\section{Dovepress}

The manuscript management system is completely online and includes a very quick and fair peer-review system, which is all easy to use. Visit http://www.dovepress.com/testimonials.php to read real quotes from published authors. 